\title{
Spontaneous right cervicothoracic lymphocoele
}

\author{
Francis J. Podbielski, MD, ${ }^{a}$ Heron E. Rodriguez, MD, ${ }^{\mathrm{b}}$ Risal S. Djohan, MD, ${ }^{\mathrm{b}}$ Mark M. Connolly, MD, ${ }^{\mathrm{b}}$ and \\ A. Alan Conlan, MD, ${ }^{a}$ Chicago, III, and Worcester, Mass
}

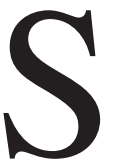

ubcutaneous accumulation of lymphatic fluid usually results from disruption of lymph channels or outflow obstruction. The cause of chylothorax, a subset of this disorder, has been classified by DeMeester ${ }^{1}$ into congenital, traumatic, diagnostic procedures (iatrogenic), and neoplastic categories. The primary imaging modalities to assess lymphocoeles are computed tomography and magnetic resonance imaging. Lymphoscintigraphy and lymphangiography are used occasionally to delineate the course of lymph flow. Isosulphan blue injection is used to aid in the localization of disrupted lymphatic channels during operative repair.

Treatment of groin and abdominal lymphocoeles consists of observation only, aspiration (often repeated), instillation of sclerosing agents, irradiation, and surgical closure-excision. Most cases of mediastinal lymphocoele can be attributed to iatrogenic ${ }^{2}$ or accidental ${ }^{3}$ trauma, although large cysts of the thoracic duct in the mediastinum have been reported without a clear cause. ${ }^{4}$ Even less frequently, these cysts manifest themselves as a supraclavicular mass confined to or predominantly located in the neck. 5,6 Cysts of the right lymphatic system are extremely rare. Only one case of a mediastinal lymphocoele has been reported that was thought to arise from the right lymphatic system. ${ }^{7}$

\section{Clinical Summary}

The patient is a 32-year-old otherwise healthy woman who noticed the abrupt onset of a large right cervical-supraclavicular fossa fullness. The only recent physical exertion she could recall was a vigorous and aerobic (ie, noncontact) workout 4 days before onset. She denied a history of pain immediately after the activity or during the following days. The area measured $7.0 \times 5.0 \mathrm{~cm}$, was not tender, was not fluctuant, and had no overlying erythema. The patient denied previous surgery or other trauma to the neck or chest. There was no abdominal distention.

Computed tomography and magnetic resonance imaging showed a right supraclavicular and unilateral right paratracheal

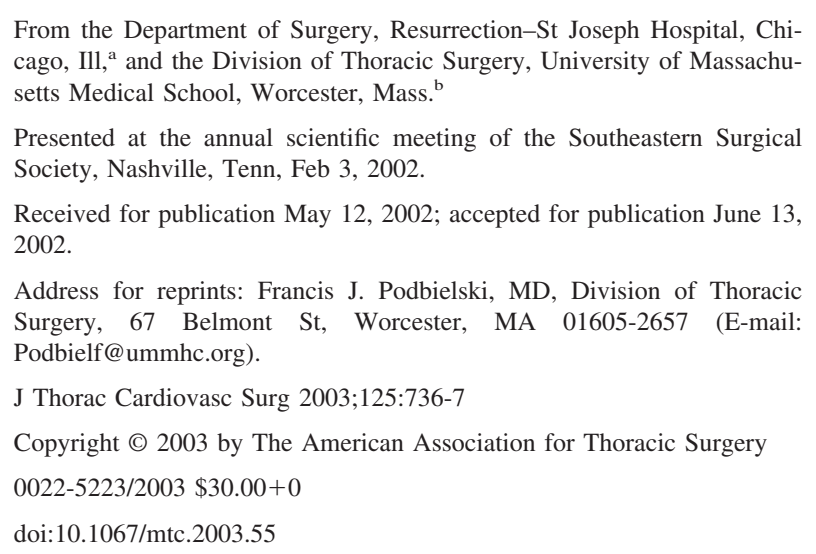

fluid collection in the superior mediastinum between the brachiocephalic artery and the superior vena cava (Figure 1). This fluid collection continued cranially posterior to the carotid sheath and lateral to the scalene muscles into the right neck (Figure 2). There was no pleural effusion, and the observed fluid collection did not cross the midline. Aspiration of the area yielded $80 \mathrm{~mL}$ of clear yellow fluid. ${ }^{8}$ Cytologic examination of the fluid showed primarily mature lymphocytes with no malignant cells. No further radiographic studies were performed, and at 1 year after injury, a smaller but persistent collection of fluid remains.

\section{Comment}

The thoracic duct transports the majority of lymph and chyle from the lower half of the body and intestines. It begins as a coalescence of smaller lymph channels that form the cisterna chyli in the abdomen. It is here that blunt abdominal trauma and malignancy can cause obstruction to lymph flow, with resultant chylous ascites. The duct ascends along the aorta (usually to its right side) through its hiatus into the right side of the chest. Sandwiched between the vertebral column and aorta, the duct crosses from the contralateral thorax at the fifth or sixth vertebral bodies. Esophageal and aortic repair-reconstruction procedures can violate the thoracic duct at this level, leading to unilateral or bilateral chylothorax. Emerging at the root of the left neck between the esophagus and the pleura, the duct might arch as high as 3 to $4 \mathrm{~cm}$ behind the carotid sheath and jugular lymphatic trunk before it terminates at the junction of the left subclavian and internal jugular veins. A valve is present in the last centimeter of the duct. This pattern of drainage explains the sporadic reports of left supraclavicular fluid collections that are eventually proven to be caused by thoracic duct cysts. In the cervical location the duct can be injured during lymphadenectomy or neck dissection and even with insertion of central venous catheters or pacemakers.

In contrast to the thoracic duct, the major lymphatic channel draining the right side of the head and right upper extremity is formed from the coalescence of the right jugular, transverse cervical, and subclavian trunks. This main right lymphatic duct is usually only 2 to $5 \mathrm{~mm}$ in length and courses along the anterior border of the scalenus anticus muscle. Its termination mirrors that of the thoracic duct by terminating posteriorly at the junction of the right internal jugular and subclavian veins. Again, a bicuspid valve is reliably found within this duct to prevent reflux of blood from the venous system. ${ }^{9}$

El Zawahry and colleagues, ${ }^{10}$ in their study of the thoracic duct and lymphovenous junction, found that major cervical lymphatic channels (unlike their abdominal counterparts) have little muscle in their walls and negligible peristalsis. This passive conveyance of lymph, although continuous, occurs mainly during the expiratory phase of the respiration. With an increase in venous pressure, valve closure prevents reflux of blood into the lymphatic system. Noncontact and nonpenetrating isolated trauma to the right-sided lym- 


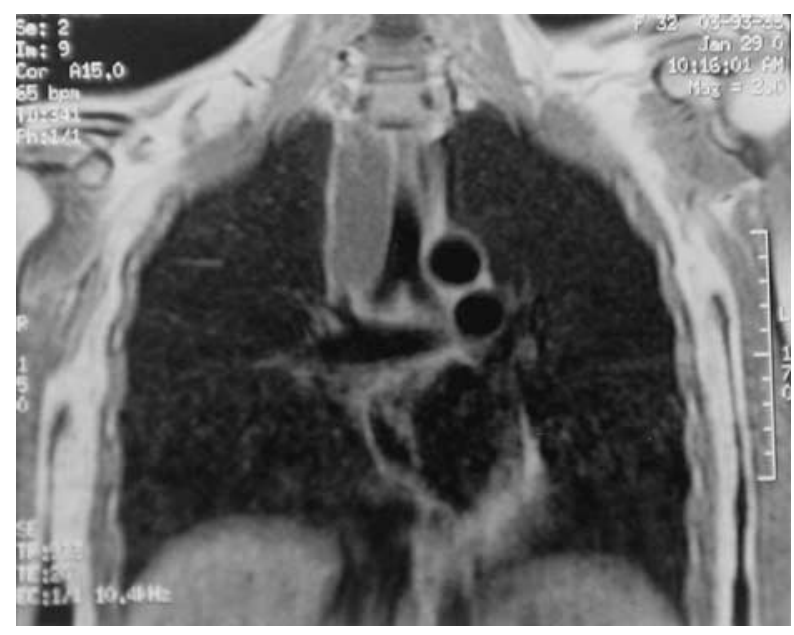

Figure 1. Magnetic resonance imaging (with gadollinium) of the thorax demonstrating a unilateral right paratracheal fluid collection in the superior mediastinum.

phatic system is extremely rare because of its short course and rich collateral drainage. In this case the distribution of fluid and the relative paucity of red blood cells in the sample is suggestive of injury near but not affecting the competence of the main right lymphatic duct valve as it drains into the subclavian vein. The postulated mechanism of injury is cervical hyperextension, resulting in injury to the duct as it courses adjacent to the medial border of the scalenus anticus muscle. The fluid distribution is consistent with injury to the right-sided system, rather than the left-sided system, given the lack of a pleural effusion and tracking of the fluid into the right, rather than the left, subclavian region.

Treatment strategies include conservative management only, which in this case seems to have been successful given the gradual clinical resolution of the lymphocoele. Surgical access to the area of injury would be difficult, requiring a low lateral cervical incision and partial resection of the medial one half to one third of the clavicle. Ligation of the duct could in theory lead to some degree of unilateral facial, cervical, and/or upper extremity edema. Given the lymph flow dysfunction in this patient and the absence of facial or extremity edema, it seems likely that adequate collateral flow is present, and thus surgical ligation would be a safe option should the lymphocoele recur.

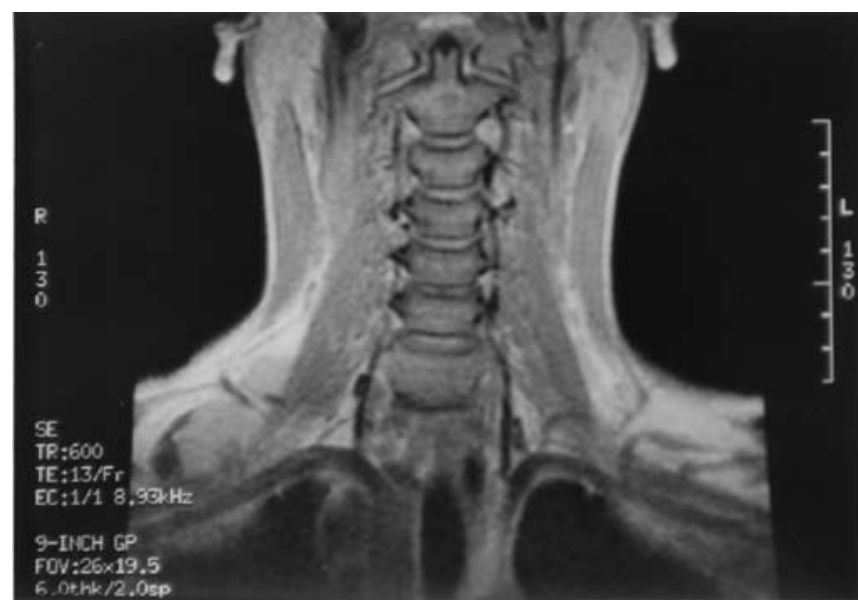

Figure 2. MRI scanning (with gadolinium) of the upper thorax showing cranial extension of the lymphocoele into the right neck posterior to the carotid sheath and lateral to the scalene muscles.

\section{References}

1. DeMeester TR. The pleura. In: Sabiston DC, Spencer EC, editors. Surgery of the chest. 4th ed. Philadelphia: WB Saunders; 1983.

2. Monk DN, Nicholson DA, Lee W, Bancewicz J. Symptomatic mediastinal lymphatic cyst after esophagectomy. Dis Esophagus. 1999;12: 82

3. Allen SJ, Koch SM, Tonnesen AS, Bowman-Howard M, Khalil K. Tracheal compression caused by traumatic thoracic duct leak. Chest. 1994;106:296-7.

4. Karajiannis A, Krueger T, Stauffer E, Ris H. Large thoracic duct cyst-a case report and review of the literature. Eur J Cardiothorac Surg. 2000;17:754-6.

5. Wax MK, Treloar ME. Thoracic duct cyst: an unusual supraclavicular mass. Head Neck. 1992;14:502-5.

6. Balestra B. A peculiar, recurrent neck swelling [in German]. Schweiz, Med Wochenschr. 1997;30:1457.

7. Muramatsu M, Tamura N, Doi Y, Dambara T, Uekusa T, Masuda S, et al. A case of mediastinal lymphatic cyst possibly originating from the right thoracic duct [in Japanese]. Nihon Kyobu Shikkan Gakkai Zasshi. 1992;30:1136-40.

8. Miller JI. Chylothorax. In: Shields TW, editor. General thoracic surgery. 4th Ed. Media, PA: Williams \& Wilkins; 1994.

9. Woodburne R. Essentials of human anatomy. 7th ed. Oxford, UK: Oxford University Press; 1983.

10. El Zawahry MD, Sayed NM, El-Awady HM, Abdel-Latif A, El-Gind M. A study of the gross, microscopic and functional anatomy of the thoracic duct and the lymph-venous junction. Int Surg. 1983;68:135-8. 